\title{
Reuses of the lonic Polymer metal composites (IPMCs) with various thicknesses
}

\author{
Shufeng Li ${ }^{1}$, a, Joanne Yip ${ }^{2}$, Gaohua Liu ${ }^{1}$ and Bowen Cheng ${ }^{1}$ \\ ${ }^{1}$ Key Laboratory of Advanced Textile Composites, School of Textile, Tianjin Polytechnic University, \\ Tianjin 300160, China; \\ ${ }^{2}$ Institute of Textiles and Clothing, Hong Kong Polytechnic University, Hung Hom, Kowloon, Hong \\ Kong, 00852. \\ alishufeng@tjpu.edu.cn
}

Keywords: IPMC, thickness, tip force, displacement.

\begin{abstract}
The IPMCs with various thicknesses were manufactured by a hot-pressed method and their actuations in terms of tip force and displacement when being reused with different treatments were investigated. Three selected treatments are no treatment, treatment in deionized water or $2 \mathrm{M} \mathrm{NaCl}$ solution. To achieve the great tip force and displacement when the IPMCs were reused, no treatment is a better way for the thin 1-film, and treatment in $\mathrm{NaCl}$ solution for the 2- and 4-films. For the thick 6-film, treatment in $2 \mathrm{M} \mathrm{NaCl}$ is appropriate to achieve the great tip force and treatment in deionized water to achieve the great displacement.
\end{abstract}

\section{Introduction}

Ionic polymer-metal composite (IPMCs) actuators are smart materials which change shape in response to an external electrical stimulus [1]. They are regarded as one of the most appropriate bionic materials for their low electrical driving forces, quick displacement velocity and bending movement and can be applied as the artificial finger[2], jellyfish[3], micro-controller[4] and robots to implement the various assignment, such as gathering the data and samples[5], operating the cells and so on.

IPMCs are composed of an ion-exchange polymer membrane, generally the perfluorinated polymers such as nafion, with two metal electrodes chemically plated on its surfaces. When an adequate potential is applied across the hydrated IPMC, it shows fast bending motion towards its positively charged surface and generate the driving forces [6]. The magnitude and speed of the bending motion of water-based IPMCs may depend on the ionic conductivity and thickness of the polymer, the structure and capacitance of the electrodes, the water content of the polymer and the mobility of the counter cations [7]. However, the nafion-based IPMC actuators have a drawback of low driving force, which limit their applications. Many researchers modified the IPMC electrodes to enhance the driving force, but it required complex and strict fabrication processes.

Recently, Sun [8] manufactured the thick IPMCs through solution casting. Furthermore, Dr. Y. H. Kim [9] proposed a simple hot-press method to adjust the thickness of the IPMCs. They approved that in air the generated tip force of the IPMCs is proportional to the number of stacked films. But how the IPMCs with various thicknesses actuate when being reused in different treatments was never studied.

In this paper, the IPMCs with various thicknesses were fabricated and their tip forces and displacements when being reused with no treatment, treatment in deionized water or $2 \mathrm{M} \mathrm{NaCl}$ solution were discussed.

\section{Experimental}

\subsection{Fabrication of IPMCs.}

Nafion®-117 from Dupont with a thickness of $0.18 \mathrm{~mm}$ was used as the ionic polymer membrane. Tetraamineplatinum( II ) chloride hydrate $\left[\mathrm{Pt}\left(\mathrm{NH}_{3}\right)_{4} \mathrm{Cl}_{2}\right](98 \%)$ was purchased from Sigma-Aldrich. All materials were used as received. 
Four IPMC specimens with various thicknesses were prepared in a hot-pressing method suggested by Kim [9]. The numbers of stacked films were 1, 2, 4, and 6, and respectively expressed as the 1-film, 2-film, 4-film and 6-film in this paper. Fabrication of the IPMCs was carried out by a two-step chemical plating method reported by our work [10].

\subsection{Measurement and characterization.}

An IPMC actuator was cut into specimens with dimensions of $6 \mathrm{~mm}$ in width and $45 \mathrm{~mm}$ in length. One edge of the IPMCs was clamped and the tip force was measured using a balance with the sensitivity of $0.0001 \mathrm{~g}$ [11]. The displacement of the IPMCs under an applied voltage was recorded by a digital camera. Reuses of the IPMCs were performed at $4.5 \mathrm{~V}$ of DC power for $1 \mathrm{~min}$. Before each use, the IPMCs were handled respectively with no treatment, treatment in deionized water or $2 \mathrm{M}$ $\mathrm{NaCl}$ solution for $5 \mathrm{~min}$.

\section{Results and discussion}

\subsection{Tip forces of the IPMCs with various thicknesses.}

Fig. 1 shows the maximum tip force losses of the IPMCs with various thicknesses when being reused in different ways. It is found that reuses of the IPMCs with three treatments mentioned all lead to the decrease in maximum tip force. When being reused with no treatment, for the first five times, the 1- and 4-films decrease more than the 2- and 6-films. After that, the maximum tip forces of the four IPMCs decrease in order of the increasing thickness. The 1-film decreases the least, and the 6-film decreases the most (Fig. 1a). After being reused ten times, the thick 4- and 6-films decreases respectively to 12 and 3 percent of their original maximum tip forces; whereas the thin 1- and 2-films still retain about 33 percent.

Compared with no treatment, treatment in deionized water results in a larger decrease in maximum tip force except the 6-film in Fig. 1b. The 6-film still maintains 90 percent of the original after being reused four times, 55 percent after being reused eight times and finally decreases to 1 percent.

In the case of treatment in $\mathrm{NaCl}$ solution (Fig. 1c), after being reused four times, the four IPMCs obviously show a lower decrease in maximum tip force. The 6-film shows the lowest decrease and maintains 90 percent, the 4-film 80 percent and the 1- and 2-films close to 60 percent. After being reused ten times, the remained maximum tip forces of the four IPMCs ascend in order of the increasing thickness. The 1-, 2-, 4- and 6-films respectively show 9, 22, 31 and 46 percent.

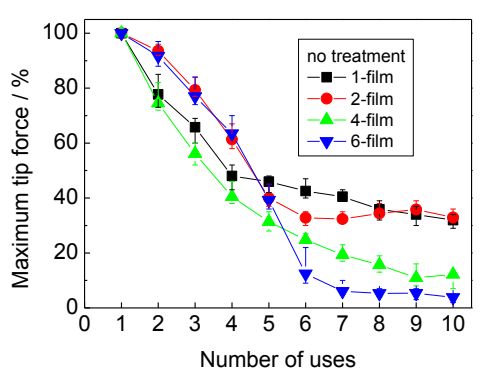

(a)

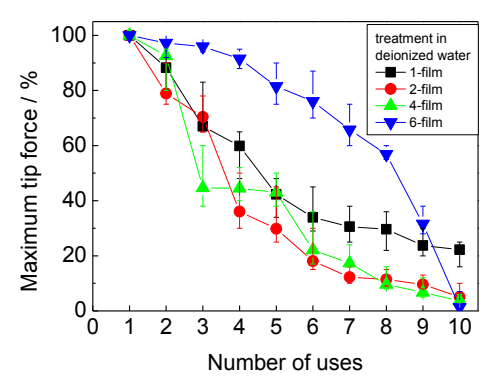

(b)

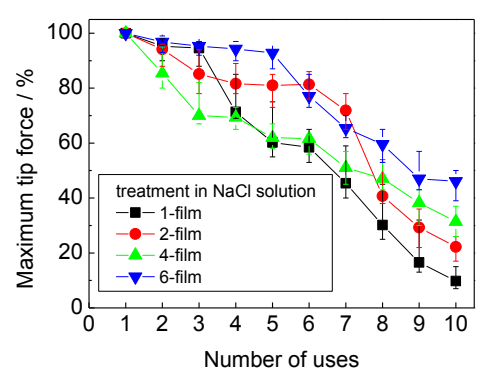

(c)

Fig. 1 Maximum tip force losses of the IPMCs with various thicknesses when being reused in different ways. (a) no treatment; (b) treatment in deionized water;

(c) treatment in $2 \mathrm{M} \mathrm{NaCl}$ solution

In conclusion, to achieve the great maximum tip force, the thin 1-film can be reused with no treatment and the thick 2-, 4- and 6-films are suggested to be reused in $\mathrm{NaCl}$ solution. This may be explained by that the thick IPMCs, which possess the long ionic migration channel, require both the hydrated cations and water more complemented. 


\subsection{Displacement of the IPMCs with various thicknesses}

Fig. 2 shows the maximum displacement losses for the IPMCs with various thicknesses when being reused in different methods. Three treatments all show a decrease in maximum displacement. When being reused with no treatment, the 1-film slowly decreases in maximum displacement and after being reused ten times still retains more than 80 percent of the original (Fig. 2a). However, the 2-, 4- and 6-films finally decrease to about 10 percent.

Compared with no treatment, treatment in deionized water (Fig. 2b) demonstrates a lower decrease in maximum displacement for the 2-, 4- and 6-films. Especially for the 6-film, after being reused five times the 6 film shows 58 percent of the original maximum displacement, evidently higher than 10 percent with no treatment. After being reused ten times, the 6-film still retains 19 percent, and the 2and 4-films decrease to about 10 percent, close to that with no treatment. The 1 -film finally declines to 10 percent, much lower than that with no treatment.

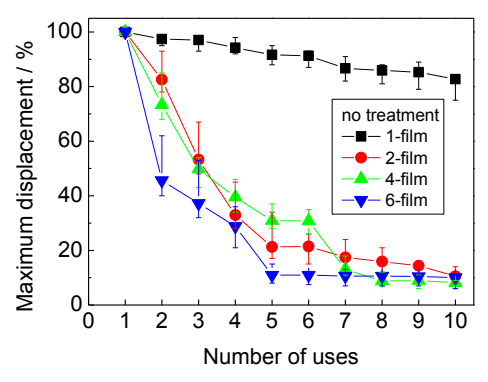

(a)

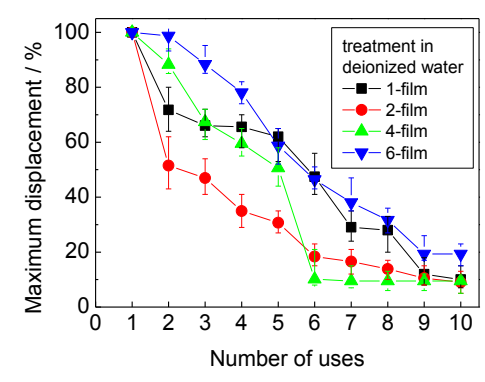

(b)

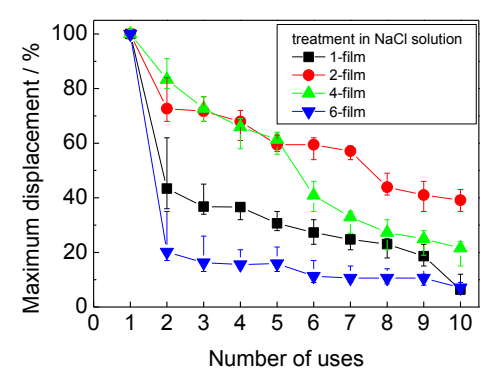

(c)

Fig. 2 Maximum displacement losses of the IPMCs with various thicknesses when being reused in different ways. (a) no treatment; (b) treatment in deionized water;

(c) treatment in $2 \mathrm{M} \mathrm{NaCl}$ solution

In comparison with no treatment and treatment in deionized water, treatment in $2 \mathrm{M} \mathrm{NaCl}$ solution results in a greater decrease in maximum displacement for the 1- and 6-films and lower decrease for the 2- and 4-films (Fig. 2c). When being reused twice, the 1-, 2-, 4- and 6-films respectively show the maximum displacement of 43, 72, 83 and 20 percent. However, after being reused ten times, the 2-film retains 40 percent and the 4-film 21 percent, the 1- and 6-films close to 10 percent.

These experimental results illustrate that the reusing types influence the maximum displacements of the IPMCs with various thicknesses. In order to obtain the greater maximum displacements, the 1-film should be reused with no treatment, the 2- and 4-films with treatment in $\mathrm{NaCl}$ solution, and the 6-film with treatment in deionized water. These may be explained by that the hydrated cations have to be supplemented when the thickness increases. But when the thickness exceeds four films, water, in place of the hydrated cations, becomes the key to maintain the displacement.

\section{Summary}

The IPMCs with various thicknesses were fabricated and effects of reuses of IPMCs with no treatment, treatment in deionized water and $\mathrm{NaCl}$ solution on the maximum tip forces and displacements were discussed. For the thick IPMCs, to maintain the greater maximum tip force, complementation of both the hydrated cations and water by treatment in $\mathrm{NaCl}$ solution is necessary; to achieve the greater maximum displacement, the deionized water plays a more important pole than the $\mathrm{NaCl}$ solution. Our experimental results are very interesting and instructive to optimize the actuations. 


\section{Acknowledgements}

This work is supported by the National Natural Science Foundation of China (No. 51603144), College Science \& Technology Fund in Tianjin City of China (No. 20140304), and Innovation Technology Fund (No. ITS/027/09).

\section{References}

[1]. Choonghee Jo, David Pugal, Il-Kwon Oh. Recent advances in ionic polymer-metal composite actuators and their modeling and applications[J]. Progress in Polymer Science. Vol. 38(2013), p. 1037-1066.

[2]. Kumar Krishen. Space applications for ionic polymer-metal composite sensors, actuators, and artificial muscles[J]. Acta Astronautica. Vol. 64(2009) No. 11-12, p. 1160-1166.

[3]. Yeom Sung-Weon, Oh Il-Kwon. A biomimetic jellyfish robot based on ionic polymer metal composite actuators[J]. Smart Materials \& Structures. Vol. 18(2009)2009, p. 085002 (10pp).

[4]. Cho SM, Lee DW. A biomimetric micro-collector based on an ionic polymer metal composite[J]. Microelectronic Engineering. Vol. 86(2009), p. 916-919.

[5]. Chung RJ, Chin TS, Chen LC, Hsieh MF. Preparation of gradually componential metal electrode on solution-casted Nafion membrane[J]. Biomolecular Engineering. Vol. 24(2007) No. 5, p. 434-437.

[6]. Mohsen Shahinpoor. Ionic Polymer-Conductor Composites As Biomimetic Sensors, Robotic Actuators and Artificial Muscles-A Review[J]. Electrochimica Acta. Vol. 48(2003) No. 14-16, p. 2343-2353.

[7]. Hong Wangyujue, Meis Catherine, Heflin James R, et al. Evidence of counterion migration in ionic polymer actuators via investigation of electromechanical performance $[\mathrm{J}]$. Sensors \& Actuators B Chemical. Vol. 205(2014), p. 371-376.

[8]. Ma Chia-Hung, Yu T.Leon, Lin Hsiu-Li, et al. Morphology and properties of Nafion membranes prepared by solution casting [J]. Polymer. Vol. 50(2009), p: 1764-1777.

[9]. Lee Sang Jun, Han Ma Jae, Kim Seong Jun, et al. A new fabrication method for IPMC actuators and application to artificial fingers[J]. Smart Materials \& Structures. Vol.15(2006), p. 1217-1224.

[10]. Yip Joanne, Li Shufeng, Wing Hang Choy, et al. Experimentally validated improvement of IPMC performance through alternation of pretreatment and electroless plating processes [J]. Smart Material \& Structures. Vol.20(2011) No. 1, p. 015009.

[11]. Lian Huiqin, Qian Weizhong, Estevez Luis, et al. Enhanced actuation in functionalized carbon nanotube-Nafion composites [J]. Sensors and Actuaors B Chemical. Vol. 156(2011), p. 187-193. 